\title{
Comment about nephrotic syndrome in association with strongyloidiasis
}

\author{
Gioacchino Li Cavoli • Calogera Tortorici • \\ Luisa Bono • Angelo Ferrantelli • \\ Carlo Giammarresi · Ugo Rotolo
}

Received: 17 December 2010/ Accepted: 21 March 2011/Published online: 6 April 2011

(C) Japanese Society of Nephrology 2011

\section{To the Editor}

We read with interest the recent work: "Minimal change nephrotic syndrome in a patient with strongyloidiasis" [1] where Dr. Miyzaki and colleagues quote 15 reported cases of nephropathy associated with Strongyloides stercoralis (Ss). We would like to add to this list another case that we reported in 2007 regarding this topic [2]. A 25-year-old male, born in Ecuador and living in Italy from 3 years of age, developed fever, vomiting, malnutrition, abdominal pain, watery diarrhea, dehydration with arterial hypertension and edema in both lower extremities. On admission, laboratory tests showed proteinuria (4 g/day), hypoalbuminemia $(1.9 \mathrm{~g} / \mathrm{dl})$, hypercholesterolemia, eosinophilia and low platelets. Renal and liver function tests, serum immunoglobulin and complement, antinuclear antibodies, ANCA were unremarkable; HAV, HBV, HCV, HIV, VDRL, BK detection and fecal and urine cultures were negative. Screening stool for rhabditiform Ss larvae was positive. Hemoculture was positive for Escherichia coli. The renal biopsy specimen contained 32 glomeruli under light microscopy examination and all had a normal appearance. No vascular or tubularinterstitial lesions were seen. Immunofluorescent studies were negative for IgA, IgG, IgM, light chains, C1q, C3, C4 and fibrinogen. The electron microscopic examination showed disappearance of slight diaphragms and moderate fusion of foot processes of glomerular epithelial cells, associated with microvillous degeneration and sometimes with a tortuous course of the basement membrane. We made a diagnosis of minimal change disease. The patient was treated with prednisone ( $1 \mathrm{mg} / \mathrm{kg} /$ day), sulfamethoxazole-trimethoprim (800-160 $\mathrm{mg}$ twice a day) and albendazole (400 mg twice a day for 3 days); sepsis cleared up quickly and the patient was discharged; however, 3 months later he was admitted again because of acute renal failure, diarrhea and nephrotic syndrome. We detected rhabditiform $S s$ larvae and IgG anti-Ss ( $283 \mathrm{UI} / \mathrm{ml})$. The patient was successfully treated with ivermectin; screening for rhabditiform Ss larvae and $\mathrm{IgG}$ anti-Ss became negative with recovery of normal renal function. Six months later the patient did not show any sign of parasitic infection but there was proteinuria $(1 \mathrm{~g} /$ day $)$ without any other sign of nephrotic syndrome. Afterwards the patient was lost to follow-up.

Conflict of interest All the authors have declared no competing interest.

\section{References}

1. Miyazaki M, Tamura M, Kabashima N, Serino R, Shibata T, Miyamoto T, et al. Minimal change nephrotic syndrome in a patient with strongyloidiasis. Clin Exp Nephrol. 2010;14:367-71.

2. Rotolo U, Scarlata F, Giordano S, Tortorici C, Bono L, Coglitore $\mathrm{M}$, et al. Nephrotic syndrome and Gram-negative sepsis in a patient with strongyloidiasis: a case report. Infez Med. 2007; 1:59-62.

G. Li Cavoli $(\bowtie) \cdot$ C. Tortorici · L. Bono · A. Ferrantelli ·

C. Giammarresi - U. Rotolo

Nephrology and Dialysis Division, Civic and Di Cristina

Hospital, via Francesco Cilea 43, 90144 Palermo, Italy

e-mail: gioacchinolicavoli@libero.it 